\title{
Hydrogen Stark broadening calculations in white dwarf atmosphere conditions
}

\author{
J. Rosato, I. Hannachi and R. Stamm \\ Laboratoire PIIM, UMR 7345 Aix-Marseille Université / CNRS, F-13397 \\ Marseille Cedex 20, France \\ (E-mail: joel.rosato@univ-amu.fr)
}

Received: July 26, 2019; Accepted: August 22, 2019

\begin{abstract}
We discuss a selection of problems involved in hydrogen Stark broadening calculations in white dwarf atmosphere conditions. The ion dynamics issue, the description of quadrupolar interactions, and the Zeeman splitting due to strong magnetic fields are successively considered. Calculations of the $\mathrm{H} \beta$ line shape are performed as an illustration.
\end{abstract}

Key words: White dwarfs - Stark effect - Zeeman effect

\section{Introduction}

Studies of white dwarf atmospheres have shown that the majority of white dwarfs have an atmosphere of pure hydrogen as a result of gravitational setting, which removes helium and heavier elements from the atmosphere and moves them towards inner layers [Fontaine \& Michaud (1979); Rohrmann (2001)]. These atmospheres can be considered as hydrogen plasmas, which are similar to some that can be created in laboratory experiments [Parigger et al. (2018)]. Such white dwarfs are classified as being of the DA type due to the strong hydrogen absorption lines they present. The electron density in a white dwarf atmosphere is high enough (up to $10^{17} \mathrm{~cm}^{-3}$, and higher) so that the line shapes are dominated by Stark broadening and, hence, can serve as a probe of the electron density $N_{e}$. In this work, we give a review of specific issues which are involved in the modeling of Stark broadening in white dwarf atmospheres. Calculations of the $\mathrm{H} \beta$ line shape are performed as an illustration.

\section{Stark broadening with ion dynamics}

We first give a summary of the line broadening formalism involved in line shape calculations. We focus on the spectral profile of a single line, without considering the contribution of continuum radiation; the latter can be addressed by using a dedicated model for the atomic populations, such as in [Tremblay \& Bergeron (2009)]. Consider an atom that emits or absorbs a photon (for the sake of clarity in discussions, it will always be referred to as the "emitter") in the presence of charged particles located at its vicinity. The electric field they generate modifies 
the energy level structure of the emitter (Stark effect), and the resulting spectral lines are broader. The spectrum of a Stark-broadened line is proportional to the Fourier transform of the dipole autocorrelation function:

$$
I(\omega) \propto \operatorname{Re} \int_{0}^{\infty} d t\{\operatorname{Tr}[\rho \boldsymbol{d}(0) \cdot \boldsymbol{d}(t)]\} e^{i \omega t} .
$$

Here, $\boldsymbol{d}(t)$ denotes the atomic dipole operator expressed in the Heisenberg picture, $\rho$ is the density operator, $\operatorname{Tr}(\ldots)$ denotes a trace over the atomic states and the brackets $\{\ldots\}$ correspond to an average over the perturbers. The dipole at time $t$ is related to its initial value through $\boldsymbol{d}(t)=U^{\dagger}(t) \boldsymbol{d}(0) U(t)$, where $U(t) \equiv U(t, 0)$ is the evolution operator from time 0 to $t$. The latter obeys the time-dependent Schrödinger equation:

$$
i \hbar \frac{d U}{d t}(t)=\left[H_{0}+V(t)\right] U(t) .
$$

Here, $H_{0}$ is the Hamiltonian corresponding to the unperturbed atom and $V(t)=$ $-\boldsymbol{d} \cdot \boldsymbol{E}(t)$ is the Stark effect term; $\boldsymbol{E}(t)$ is the electric field generated at the emitter's location by the perturbers. In the framework of the so-called "standard model", the ions are assumed motionless and the electrons are described within a collisional picture in terms of a relaxation ("collision") operator $K_{e}$. In practice, these two assumptions yield the substitution $-\boldsymbol{d} \cdot \boldsymbol{E}(t) \rightarrow-\boldsymbol{d} \cdot \boldsymbol{E}_{i}(0)-i K_{e}$ in the Stark effect term; the Schrödinger equation has an exponential solution and the line shape is obtained from a matrix inversion (e.g. Griem (1974) for details). The standard model provides a good estimate of the line broadening but it can be inaccurate in regimes such that the ions move significantly during the characteristic decorrelation time for the dipole (usually referred to as the "timeof-interest" $t_{i}$; it is of the order of the inverse line width). An illustration of this inaccuracy is given in Figure 1. The spectral profile of $\mathrm{H} \beta$ has been calculated assuming a pure and completely ionized hydrogen plasma, of density $N_{e}=$ $N_{i}=10^{17} \mathrm{~cm}^{-3}$ and temperature $T_{e}=T_{i}=1 \mathrm{eV}$. These values correspond to white dwarf atmosphere conditions. For the sake of clarity, the broadening due to electrons has not been retained in the calculations. The calculation with account of ion dynamics has been done using a computer simulation technique, developed previously for tokamak plasma spectroscopy applications [Rosato et al. (2009)]. As can be seen in the figure, the ion dynamics yields a spectrum larger than that expected from a static ion model. This result is in agreement with early studies of ion dynamics [e.g. Stamm et al. (1986)]; a noticeable result is that the dip at the center is less pronounced. A criterion for the static approximation for ions to hold is that the collision time $\tau_{c}=r_{0} / v\left(\right.$ with $r_{0} \sim N_{i}^{-1 / 3}$ and $v \sim \sqrt{2 T_{i} / m_{i}}$ ) must be larger than the time-of-interest. In the conditions considered here, the ratio $t_{i} / \tau_{c}$ is of the order of several tens of percents, which indicates that ion dynamics effects cannot be neglected. 


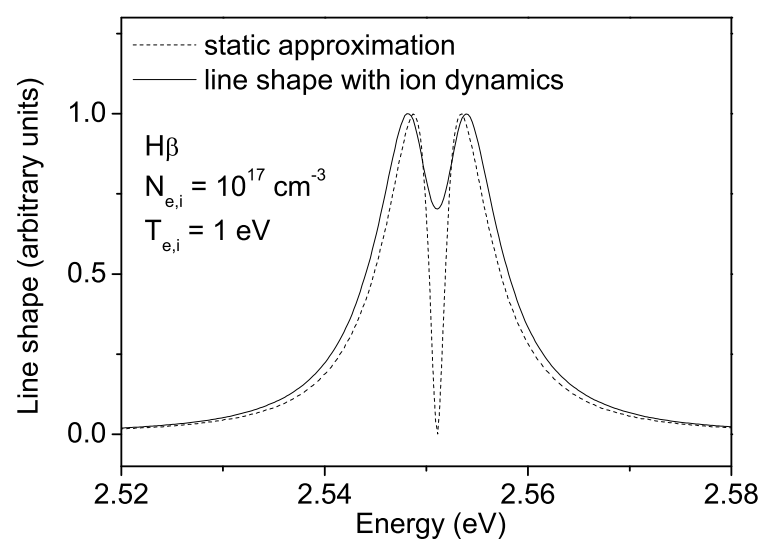

Figure 1. Spectral profile of $\mathrm{H} \beta$ calculated in white dwarf atmosphere conditions, assuming a static model for ions and retaining the ion dynamics during the time-of-interest. The ion dynamics results in an additional broadening of the line. In particular, the dip present at the center of the line is less pronounced.

\section{Quadrupolar interaction}

The Stark effect Hamiltonian $V=-\boldsymbol{d} \cdot \boldsymbol{E}$ corresponds to the first-order term in a multipole expansion; in dense media, the mean distance between an emitter and a perturber can be small enough so that higher-order terms need to be retained. Other-than-dipole interactions can result in an asymmetry of the spectral lines [Djurović et al. (2009); Halenka et al. (2015); Gomez et al. (2016)]. Figure 2 shows an illustration of such an asymmetry in white dwarf atmosphere conditions. The $\mathrm{H} \beta$ line shape has been calculated with account of the quadrupolar interaction, assuming the same plasma parameters as in the previous section. The quadrupolar interaction is described through an additional term in the Hamiltonian, $V_{Q}=-(1 / 3) \boldsymbol{Q}: \boldsymbol{\nabla} \boldsymbol{E}$, where the quadrupolar moment is defined as $\boldsymbol{Q}=-e\left(3 \boldsymbol{r} \boldsymbol{r}-r^{2} \boldsymbol{I}\right) / 2$ with $\boldsymbol{r}$ being the position of the atomic electron and $\boldsymbol{I}$ being the identity matrix. The broadening due to electrons has not been retained here, again for clarity purposes. As can be seen in the figure, the blue component of the line is higher than the red component. This asymmetry is all the more pronounced that the density is high. Specific investigations using line shape codes have been carried out recently in order to address this effect [SLSP workshop; http://plasma-gate.weizmann.ac.il/slsp/] and calculations are still underway. In particular, a pending issue is the relative importance of multipolar interactions with respect to the quadratic Stark effect (namely: the coupling between states of different principal quantum numbers), which is important in high density 
regimes and also yields an asymmetry. These two effects must be treated on an equal footing in calculations in order to have an accurate description of spectra.

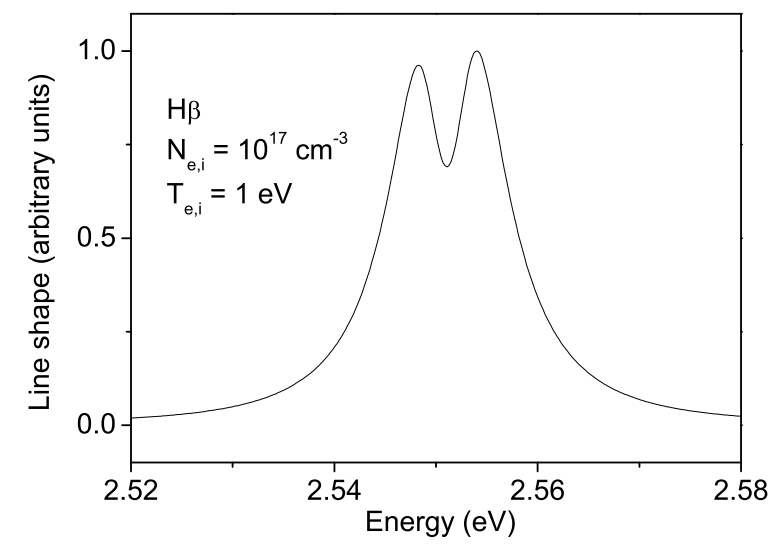

Figure 2. The quadrupolar interaction yields an asymmetry. Here, the $\mathrm{H} \beta$ line shape has been calculated with the same conditions as in Fig. 1.

\section{Zeeman effect}

A significant amount of white dwarf spectra exhibit lines with a triplet structure, which is a feature of the presence of strong magnetic fields [Külebi et al. (2009); Kepler et al. (2013)]. Simple estimates based on the separation between the components indicate that the magnetic field can attain several kiloteslas. The combined action of electric and magnetic fields on lines has recently been addressed in white dwarf atmosphere conditions [Rosato et al. (2017); Kieu et al. (2017)]. A pending issue is the description of the overlapping and the mixing between two consecutive lines (e.g., $\mathrm{H} \beta$ and $\mathrm{H} \gamma$ ) in regimes such that the Zeeman spitting is comparable to the separation between the lines. A proper description of a spectrum should account both for the linear Zeeman perturbation $-\boldsymbol{\mu} \cdot \boldsymbol{B}$ (with $\boldsymbol{\mu}$ being the atomic magnetic moment) and the quadratic perturbation $e^{2}(\boldsymbol{B} \times \boldsymbol{r})^{2} / 8 m_{e}$ [Garstang (1977)]. Figure 3 shows an example where such a situation occurs. The $\mathrm{H} \beta$ line shape has been calculated assuming the same plasma conditions as above. A value of $2 \mathrm{kT}$ has been taken for the magnetic field and the observation angle $\theta$ has been set equal to $90^{\circ}$. The spectrum presents many components, which is a feature of the quadratic Zeeman effect. The plot in dashed line corresponds to a calculation of $\mathrm{H} \beta$ neglecting the energy level mixing and the solid line corresponds to a calculation where mixing with the $n=5$ 
level is retained. As can be seen, there is an overall shift of the spectrum towards the red direction. This shift is a feature of the repulsion between energy levels due to an external perturbation; it is expected from basic time-independent perturbation theory. A noticeable result is that not all components are shifted with the same amplitude. Calculations are presently underway in order to better characterize this effect.

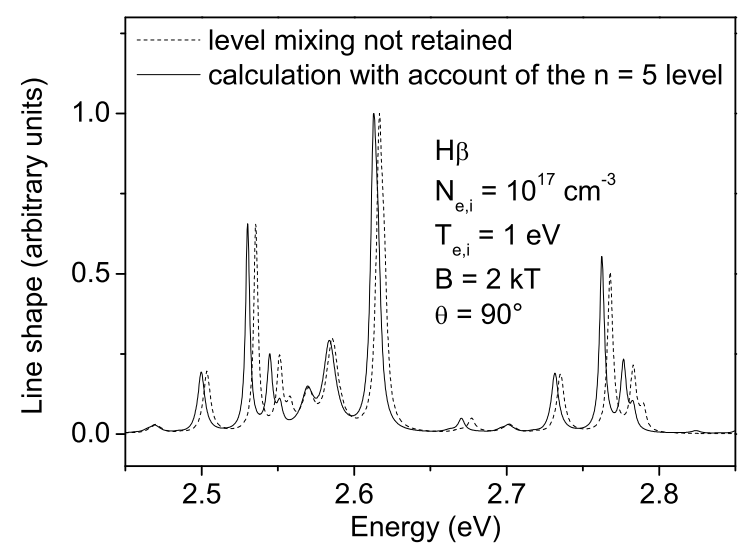

Figure 3. Some white dwarfs have a strong magnetic field; as a result, the spectral lines are subject to both linear and quadratic Zeeman effects. The quadratic Zeeman effect can induce a mixing between energy levels of different principal quantum number, which must be retained in a calculation if accuracy is seeked for. Here, the figure shows an example where such a situation occurs. The calculation neglecting energy level mixing (dashed line) yields an overall shift of the spectrum. Not all components are shifted with the same amplitude.

\section{Conclusion}

We have identified several problems involved in the modeling of hydrogen Stark line shapes in conditions relevant to white dwarf atmospheres. A line can be broader due to ion dynamics effects, and, on the other hand, it can be asymmetric due to quadrupolar interactions. These two effects must be accounted for in models if accuracy is seeked for in a spectroscopic diagnostic. An additional issue that must be addressed is the presence of quadratic Zeeman effect and its influence on energy level mixing. The $\mathrm{H} \beta$ calculations we have done indicate a significant shift of the overall spectrum. New calculations are presently ongoing in order to better quantify this effect. An additional issue that will be addressed 
is the modification of the particle trajectories due to the magnetic field, and how this alters spectral line shapes. New investigations will be done along the lines of previous works [Rosato et al. (2018); Alexiou (2019)].

Acknowledgements. This work has been carried out within the framework of the French Research Federation for Magnetic Fusion Studies.

\section{References}

Alexiou, S. 2019, Atoms, 7, 52

Djurović, S. et al. 2009, Phys. Rev. E, 79, 046402

Fontaine, G. \& Michaud, G. 1979, Astrophys. J., 231, 826

Garstang, R. H. 1977, Rep. Prog. Phys., 40, 105

Gomez, T. A. et al. 2016, Phys. Rev. A, 94, 022501

Griem, H. R. 1974, Spectral Line Broadening by Plasmas, ed. A. Press (London)

Halenka, J. et al. 2015, Astrophys. J., 808, 131

Kepler, S. O. et al. 2013, Mon. Not. R. Astron. Soc., 429, 2934

Kieu, N. et al. 2017, Atoms, 5, 44

Külebi, B. et al. 2009, Astron. Astrophys., 506, 1341

Parigger, C. G. et al. 2018, Atoms, 6, 36

Rohrmann, R. D. 2001, Mon. Not. R. Astron. Soc., 323, 699

Rosato, J., Ferri, S., \& Stamm, R. 2018, Atoms, 6, 12

Rosato, J. et al. 2009, Phys. Rev. E, 79, 046408

Rosato, J. et al. 2017, Atoms, 5, 36

Stamm, R. et al. 1986, Phys. Rev. A, 34, 4144

Tremblay, P.-E. \& Bergeron, P. 2009, Astrophys. J., 696, 1755 